\title{
Footstep Power Generation Using Piezoelectric Sensor
}

\author{
Adeel Ali ${ }^{1}$, Usama Khan ${ }^{1}$, Md. Omair Ahmad ${ }^{1}$, Asfia Aziz ${ }^{1,} \mathrm{Neha}^{1}$, \\ \{adeel.ali.2222@gmail.com\} \\ ${ }^{1}$ School of Engineering Science and Technology (SEST) Jamia Hamdard \\ New Delhi, India
}

\begin{abstract}
In today's world, the need for power is increasing at a very fast pace. The problem faced by lack of power may have a number of solutions and the generation of power using footsteps is among one of the infinite ways of producing renewable resources of energy. In this power generation method power is generated using force applied during walking, the piezoelectric sensors are used as the source of generation of power. In this model, the series connection of the piezoelectric is further connected to a circuit that helps in storing the power generated. This energy can be generated while walking on certain arrangements like footpaths, stairs, stations, etc. and can be generated in any densely populated area for maximum efficiency.
\end{abstract}

Keywords: Piezoelectric Sensor, Piezoelectricity, Pressure Sensor, Footstep Power Generation

\section{Introduction}

Piezoelectric Effect is the ability of some materials to produce an electric charge in response to applied pressure. The word Piezoelectric is derived from the Greek piezein, which means to squeeze or press, and piezo, which is a Greek word for "push" [1]. Materials which show direct piezoelectric effect follows a reverse behaviour meaning when stress is applied to these materials, they produce electricity (Direct Piezoelectric Effect) and in reverse conditions, when electricity is applied to these materials, they produce stress. To develop a working model, we have used connected four piezoelectric sensors in series which are further connected to a complex circuit which includes 7805 Voltage Regulator IC, IN4007 Diodes, booster convertor, etc. Piezoelectric materials can be used as bending, compression or shear mode sensors. All three modes are sensitive to external force applied to the material but the highest sensitivity is awarded to the bending mode (LIAO, 2005) [2] When pressure is applied on piezo material, a charge is generated across it. Thus, it acts as an ideal Capacitor (Kiran Boby)[3]

\section{Methodology}


A working model of Footstep Power Generation is demonstrated in this project, the basic working of this model has been presented as a block diagram (Fig.1). To implement this model four piezoelectric sensor that are connected in series to increase the voltage output, this sensor generates AC voltage which is transferred to the bridge rectifier. The output voltage from the sensor can be of two types because it produces AC voltage:

Positive: When positive voltage is received then it is passed into the forward bias mode which will in turn charge the battery.

Negative: When a negative voltage is received then it is transferred to the reverse bias of the bridge rectifier.

This way no voltage loss is observed during a complete cycle. An additional resistor of 470 $\mathrm{Ohm}$ is placed in series along with a capacitor of $4.7 \mathrm{mF}$ with the LED to make the glow more noticeable.

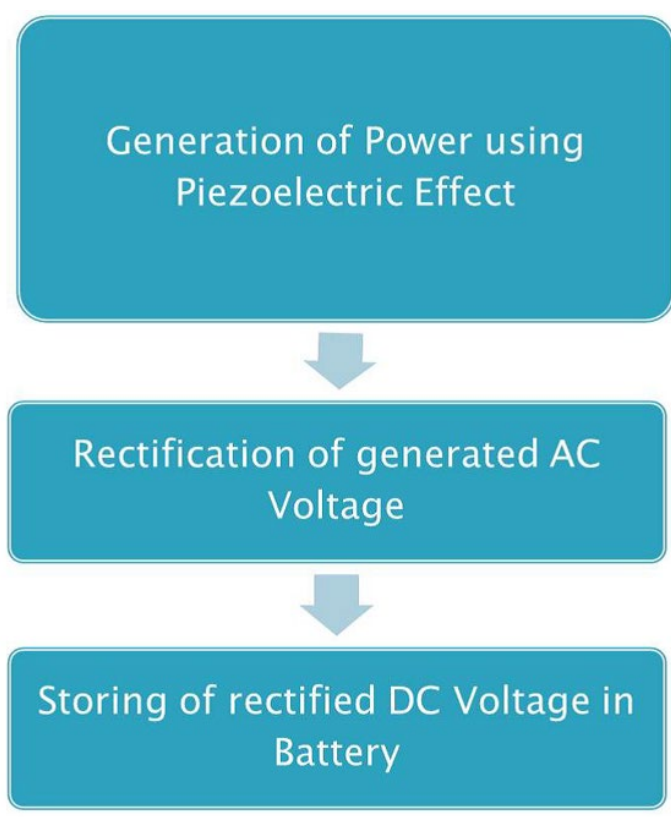

Figure 1: Block Diagram for generation of electricity

\subsection{Piezoelectric Effect}


The piezoelectric effect refers to a variation in electric polarization that is produced in some materials when pressure is applied on them. This pressure-dependent variation in polarization establishes as a measurable potential difference across the material. Referred to as the direct piezoelectric effect, this phenomenon is evident in many naturally occurring crystalline materials, including quartz, Rochelle salt, and even human bone. Engineered materials, such as lithium niobate and lead zirconate titanate (PZT), exhibit a more distinct piezoelectric effect (cosmol.co.in)[4][5] Piezoelectricity describes interconversion between electrical charge and mechanical strain. The direct piezoelectric effect is experiential as a change in surface charge density of a material in response to an external pressure (Ilias Katsouras 1)[6]

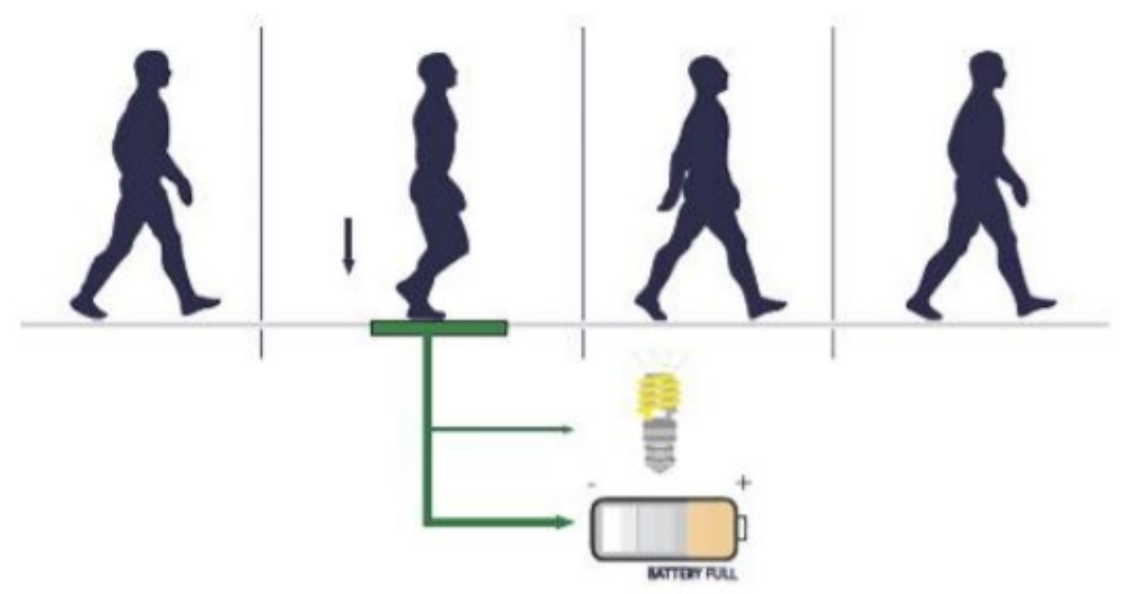

Figure 2: Schematic representation of the working model [7] 


\section{Circuit Description}

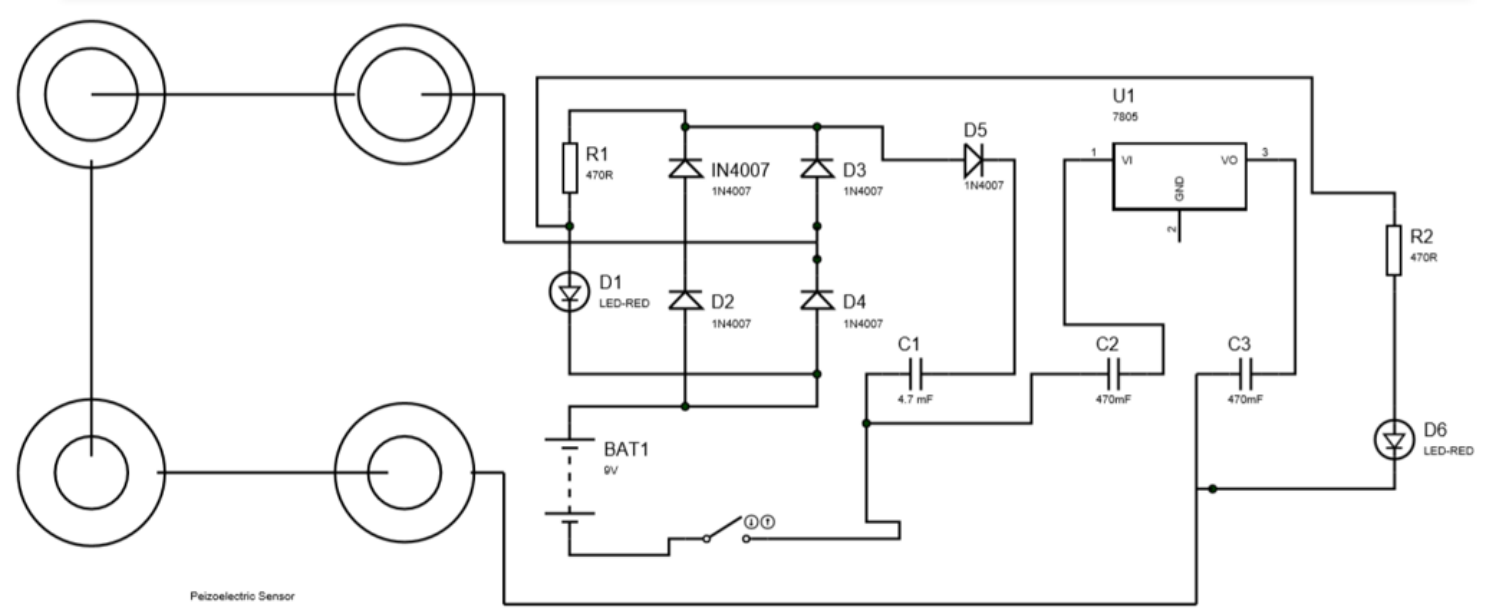

Figure 3: Circuit Diagram for Footstep Power Generation Using Piezoelectric Sensor

In the final developed model, four piezoelectric sensors are coupled in series to increases the output voltage. The voltage produced after applying force on the piezoelectric sensor is AC and therefore it travels through the bridge rectifier to avoid any loss of voltage. The positive and negative voltages are utilised to charge the rechargeable battery. A $4.7 \mathrm{uF}$ capacitor along with a $470 \Omega$ resistor is connected in series with the LED to indicate the electricity produced by applying force, this capacitor also helps in retaining the glow of electricity. Now, to further charge a device using the produced electricity we place one IN4007 diode in forward biased mode, this ensures the flow of current in only in one direction and hence minimizing current loss. For charging a mobile station we further connect this circuit to 7805 Voltage Regulator IC which can accept voltage ranging from $9 \mathrm{v}$ to $18 \mathrm{v}$ and outputs a voltage of $5 \mathrm{v}$, this charging is only enabled when the switch is turned on. Two capacitors of $470 \mathrm{uF}$ are connected on the input and output pins of 7805 Voltage Regulator IC to make the current flow smooth. To obtain a female USB A port we have connected a booster convertor which can be used to charge a device. 


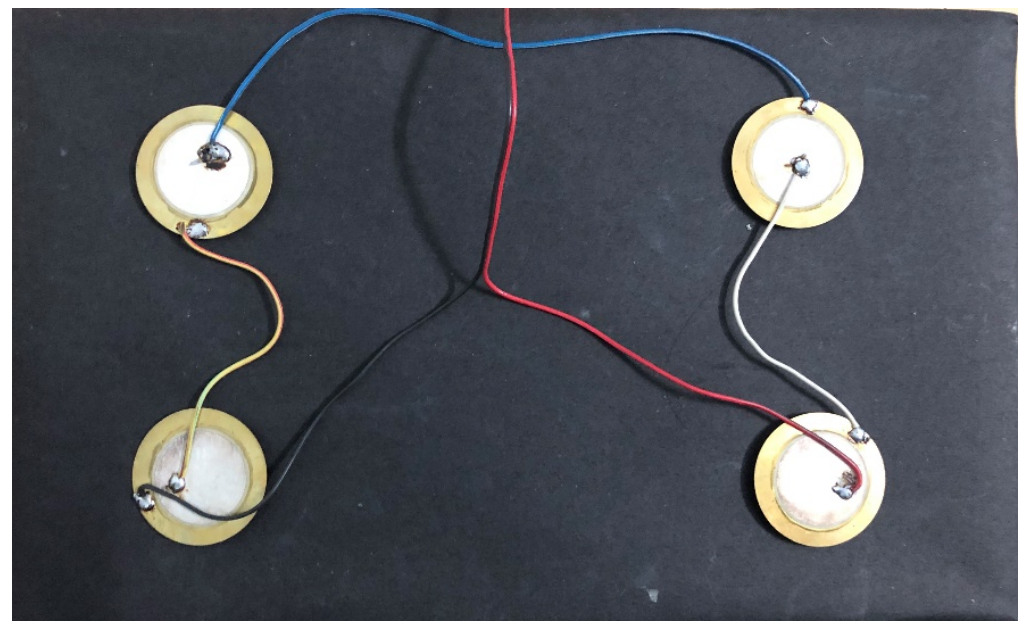

Figure 4: Piezoelectric Sensors in series connection

\section{Results}

We have successfully developed a working model to produce power by applying pressure imitating walking situations, this can also be done by physically walking on the setup of piezoelectric sensors. This model effectively demonstrates the use of power which was previously neglected, the developed model is extremely simple and highly useful. This system can output a good amount of power which can further be used for providing huge areas with electricity.

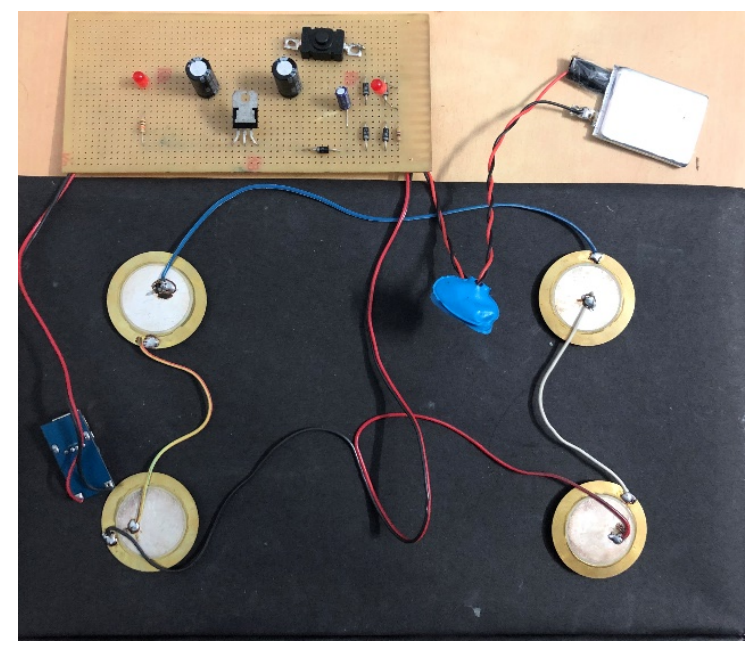

Figure 5: Developed model 


\section{Conclusion}

The project presented is a well-balanced model of energy generation and ease of setup and implementation. This model can be used as an alternative for power supply if implemented on a large scale. Further, this can be applied to areas where there is an extensive need of power conservation. The key component to this model is mechanical stress or pressure which in most scenarios occurs naturally. This piezoelectric system can be applied on large surfaces with large surface areas, once implemented the surface can be moulded back into its original shape and hence decreasing the risk of damaging the physical appearance of the place. Roads, Railway stations, Footpath's or any place that witness a large number of crowds on daily basis can be used to implement this model and hence maximising the output power.

\section{References}

[1] https://www.nanomotion.com/piezo-ceramic-motor-technology/piezoelectric-effect/

[2] T. H. NG AND W. H. LIAO: Sensitivity Analysis and Energy Harvesting for a Self-Powered Piezoelectric Sensor: First Published October 1, 2005

[3] Kiran Boby, Aleena Paul K, Anumol.C.V, Josnie Ann Thomas, Nimisha K.K Dept of EEE, MACE, Kothamangalam: Footstep Power Generation Using Piezo Electric Transducers

[4] https://www.comsol.co.in/multiphysics/piezoelectric-effect

[5] https://www.nanomotion.com/piezo-ceramic-motor-technology/piezoelectric-effect/

[6] Ilias Katsouras1,2, Kamal Asadi1 , Mengyuan Li3,4, Tim B. van Driel5 , Kasper S. Kjær5 , Dong Zhao1, Thomas Lenz1, Yun Gu5 , Paul W. M. Blom1, Dragan Damjanovic6 , Martin M. Nielsen5 and Dago M. de Leeuw1,7*: The negative piezoelectric eect of the ferroelectric polymer poly(vinylidene fluoride)

[7] Kiran Boby, Aleena Paul K, Anumol.C.V, Josnie Ann Thomas, Nimisha K.K Dept of EEE, MACE, Kothamangalam: Footstep Power Generation Using Piezo Electric Transducers 